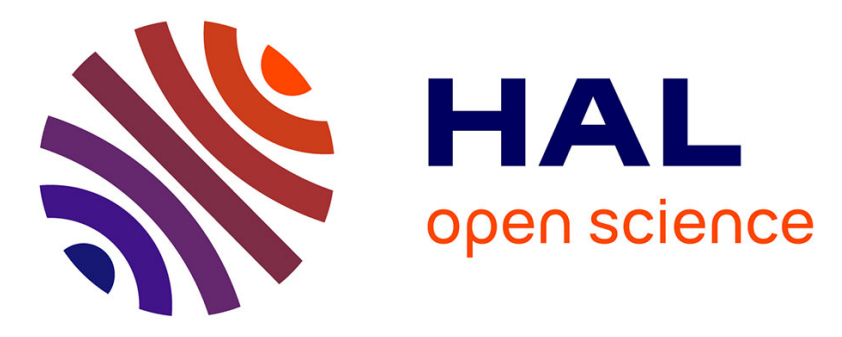

\title{
Standardization of xenon-127 and measurement of photon emission intensities
}

Marie-Christine Lépy, Matias Rodrigues, M.-C Lépy, Philippe Cassette, Xavier Mougeot, Marie-Martine M Bé

\section{- To cite this version:}

Marie-Christine Lépy, Matias Rodrigues, M.-C Lépy, Philippe Cassette, Xavier Mougeot, et al.. Standardization of xenon-127 and measurement of photon emission intensities. Applied Radiation and Isotopes, 2014, 87, pp.342 - 347. 10.1016/j.apradiso.2013.11.066 . hal-01863288

\section{HAL Id: hal-01863288 \\ https://hal.science/hal-01863288}

Submitted on 20 Feb 2020

HAL is a multi-disciplinary open access archive for the deposit and dissemination of scientific research documents, whether they are published or not. The documents may come from teaching and research institutions in France or abroad, or from public or private research centers.
L'archive ouverte pluridisciplinaire HAL, est destinée au dépôt et à la diffusion de documents scientifiques de niveau recherche, publiés ou non, émanant des établissements d'enseignement et de recherche français ou étrangers, des laboratoires publics ou privés. 


\title{
Standardization of xenon-127 and measurement of photon emission intensities
}

\author{
M. Rodrigues, M.-C. Lépy, P. Cassette ${ }^{*}$, X. Mougeot, M.M. Bé \\ CEA, LIST, Laboratoire National Henri Becquerel, Gif-sur-Yvette, F-91191, France \\ ${ }^{*}$ Corresponding author, e-mail: Philippe.cassette@cea.fr
}

\begin{abstract}
Xenon-127 was standardized by internal gas counting using three proportional counters in a differential arrangement to eliminate edge effects. The detection efficiency of the proportional counters was calculated by considering the cascade of events following the electron capture and associated gamma transitions. Activity per unit volume was measured with $0.7 \%$ relative standard uncertainty. Gamma-ray spectrometry was performed and absolute photon emission intensities were derived. This study shows that ${ }^{127} \mathrm{Xe}$ could be a surrogate for ${ }^{133} \mathrm{Xe}$ for the calibration of remote radio-xenon monitoring stations.
\end{abstract}

\section{Introduction}

The yield of production of xenon isotopes by fission of uranium and plutonium is high and, because of their chemical inertia, these radionuclides are very good tracers of civil and military nuclear activities. Xenon isotopes are used to calibrate monitoring instruments of the Comprehensive Nuclear-Test-Ban Treaty Organization (CTBTO). Monitoring of radioactive noble gas mainly concerns ${ }^{133} \mathrm{Xe},{ }^{131 \mathrm{~m}} \mathrm{Xe},{ }^{133 \mathrm{~m}} \mathrm{Xe}$ and ${ }^{135} \mathrm{Xe}$ for the purpose of determining the nature of a nuclear event. However, these isotopes have short half-lives and so are not convenient for calibrating distant monitoring stations. Even though ${ }^{127} \mathrm{Xe}$ is not the radionuclide of interest for this purpose, it can be conveniently used for the control of these devices, due to its rather long half-life (36.4 (1) d) compared to the other xenon isotopes.

\section{Decay scheme}

Xe-127 decays by electron capture to excited levels of ${ }^{127}$ I with a cascade of 5 main gamma ray emissions in the 57.6 to $375.0 \mathrm{keV}$ energy range (Figure 1), together with the characteristic X-rays of iodine. Only three transitions lead to allowed electron-capture decays, namely to the 203, 375 and $618 \mathrm{keV}$ states. Up-to-now, no other transition has been observed. The following comment can be found in (Gehrke and Helmer, 1977): "The electron-capture to the ground state is second forbidden for which the minimum $\log f t$ value is $\sim 11$ and thus the maximum intensity of the feeding branch is $\sim 0.003 \%$ ”. The same comment can be applied for the electron-capture to the first excited state of ${ }^{127}$ I since this transition is third forbidden unique.

Three measurements of relative $\gamma$-ray emission intensities and only one measurement of the absolute $\gamma$-ray emission intensities are available in the literature. The decay scheme of ${ }^{127} \mathrm{Xe}$ is therefore poorly known and must be improved for the practical use of this radionuclide for calibration purposes. 


\section{Standardization using triple gas counters}

In this study, ${ }^{127} \mathrm{Xe}$ was standardized by internal gas counting using three proportional counters in a differential arrangement to eliminate end effects. A measurement using $4 \pi \gamma$ detector can also be used (Vatin and Chauvenet, 1987) but we think that gas counting leads to more direct results.

\subsection{Source preparation}

The radioactive source was prepared from the neutron irradiation of an enriched ${ }^{126}$ Xe target. The measured sample was composed of xenon in nitrogen and contained ${ }^{125} \mathrm{I}$ as a radioactive impurity. This impurity was removed by the use of a filter made of silver wool and the radioactive purity was checked by gamma-ray spectrometry.

\subsection{Measurement method}

The internal gas counting system developed at the LNE-LNHB was described previously (Lansiart et al., 1993). It is composed of three proportional detectors with different lengths, an electronic subassembly and a gas handling equipment.

The detectors are identically constructed of stainless steel tubes of different lengths and their ends have guard rings which are in contact with the cathode. The counting gas is propane. Using these counters, three ideal detectors (without end effect) can be obtained by differential counting.

The electronic subassembly contains three counting channels and each channel is equipped with an extended dead-time circuit. The corrections due to this dead-time are made according to the live-time method. Additionally, each channel is connected to a multichannel analyzer board from a personal computer. The experimental energy spectrum given by each detector is stored in order to obtain differential spectra. The energy threshold of the counters are adjusted at $200 \mathrm{eV}$.

The gas handling equipment is used to sample the radioactive gas in a reference volume and mix it with the counting gas in the dilution volume. After homogenization, the mixture is transferred to the proportional counters.

\subsection{Detection efficiency}

The electronic re-arrangement following the electron capture and the gamma transitions both contribute to the detection efficiency of the proportional counter. The contribution of each process is evaluated hereafter. For the electron capture process, atomic rearrangements can lead to vacancies in various shells and thus, the detection efficiency is evaluated for each kind of vacancy, irrespective of the phenomenon creating this vacancy.

\section{Electron vacancy in the $N$ shells}

The binding energy of the electrons of the $\mathrm{N}$ shell is ranging from $186 \mathrm{eV}$ for the sub shell N1 to $50 \mathrm{eV}$ to the subshell N5. The fluorescence yield is negligible and thus, the higher energy Auger electron is N1-N5-X, at $136 \mathrm{eV}$, that is lower than the energy threshold of the counter. It can be concluded that the detection efficiency of the capture of $\mathrm{N}$ electrons is negligible, thus $E f_{N}=0$.

\section{Electron vacancy in the $M$ shells}


The binding energies of the electrons of the $\mathrm{M}$ shell are in the 1.07-keV to $620-\mathrm{eV}$ range for respectively M1 to M5. The fluorescence yields are from a few $10^{-4}$ to $10^{-3}$ (Mc Guire, 1972, 1974 ) and thus the X-ray emission can be neglected. The energy of Auger electrons is in the 899-eV to 233-eV energy range, respectively for M1-N2-N5 and M5-N1-X rearrangements. The Coster-Kronig electrons like M1-M2-N2 are of low energy (less than $20 \mathrm{eV}$ ), but the Auger rearrangement following the M2 vacancy will lead to detectable electrons. From this, we can assume that the detection efficiency of a vacancy in the $M$ shell, $E f_{M}$, is equal to 1.

\section{Electron vacancy in the $L$ shells}

Two kinds of rearrangements can lead to non-detection: $\mathrm{X}_{\mathrm{LN}}$ emission and LLN CosterKronig transitions with energy of the $\mathrm{N}$ electron lower than $200 \mathrm{eV}$.

The fluorescence yields of L1, L2 and L3 transitions $\omega_{L i}$ are respectively $0.043,0.084$ and 0.086 (Puri et al., 1993). The fraction of emission of $\mathrm{X}_{\mathrm{LN}}$ radiation over the total $\mathrm{XL}$ emission, $P_{X L i N}$ is $0.186,0.127$ and 0.126 respectively for L1, L2 and L3 vacancies. The average energy of these XLN radiations is between 4.37 and $5.13 \mathrm{keV}$. The probability of detection of these radiations, $P_{D}$, was calculated by Monte Carlo simulation using the PENELOPE code (Salvat et al., 2008). The simulation was done for a cylindrical counter containing a mixture of propane and nitrogen at a pressure of $300 \mathrm{hPa}$ (the amount of xenon or iodine atoms being negligible). This allows the calculation of the energy spectrum absorbed in the gas. A main photoelectric peak is observed with a distribution of lower energy events due to photon interactions in the counter stainless-steel envelope. The integral of the spectrum over $200 \mathrm{eV}$, thus corresponding to detectable events, is $P_{D}=2.8 \%$.

Among the LLN Coster-Kronig transitions, the ones leading to the emission of $\mathrm{N}$ electrons with energy lower than $200 \mathrm{eV}$ are the L1L2N1, L2L3N1, L2L3N2 and L2L3N3 transitions. The probabilities $P_{L 1 L 2 N 1}=0.052$ and $P_{L 2 L 3 N j}=0.068$, are the relative probabilities of these transitions respectively over the total Coster-Kronig transitions L1L2 and L2L3 (Chen and Crasemann, 1979).

With $f_{12}=0.18$ and $f_{23}=0.154$ being the Coster-Kronig yields, the detection efficiency of vacancy in the subshell $\mathrm{L}_{\mathrm{i}}$ is:

$$
\begin{gathered}
E f_{L 3}=1-\bar{E} f_{L 3}=1-\left(\omega_{L 3} \cdot P_{X L 3 N} \cdot\left(1-P_{D}\right)\right) \\
E f_{L 1}=1-\bar{E} f_{L 1}=1-\left(\omega_{L 1} \cdot P_{X L 1 N} \cdot\left(1-P_{D}\right)+f_{12} \cdot P_{L 1 L 2 N 1}\right) \\
E f_{L 2}=1-\bar{E} f_{L 2}=1-\left(\omega_{L 2} \cdot P_{X L 2 N} \cdot\left(1-P_{D}\right)+f_{23} \cdot P_{L 2 L 3 N j}\right)
\end{gathered}
$$

The probabilities of detection associated with subshells L1 to L3 are respectively $E f_{L 1}=0.982$, $E f_{L 2}=0.980$ and $E f_{L 3}=0.989$.

\section{Electron vacancy in the $K$ shell}

The probability of detection of KX rays is negligible but the vacancies following the X-ray emission can be detected.

The $\mathrm{X}_{\mathrm{KL} 2}$ and $\mathrm{X}_{\mathrm{KL} 3}$ emissions lead to vacancies in the L2 and L3 subshells and are thus respectively detected with the probabilities $E f_{L 2}$ and $E f_{L 3}$ previously calculated and the $X_{K L 1}$ probability being negligible. The relative probabilities of $\mathrm{X}_{\mathrm{KL} 2}$ and $\mathrm{X}_{\mathrm{KL} 3}$ over the total $\mathrm{X}_{\mathrm{K}}$ emission are respectively $P_{X K L 2}=0.290$ and $P_{X K L 3}=0.534$. The $X_{K M}$ emissions lead to vacancies in the $\mathrm{M}$ shell, and thus are detected with a probability of 1 . 
The $\mathrm{X}_{\mathrm{KN}}$ and $\mathrm{X}_{\mathrm{Ko}}$ emissions are not detected. The relative probability of these emissions over the total $\mathrm{X}_{\mathrm{K}}$ emission is $P_{X K N}+P_{X K O}=0.031$.

The detection efficiency of $K$ Auger electrons can be considered to be 1 .

The K fluorescence yield is $\omega_{K}=0.882$ and thus the total detection efficiency for a vacancy in the $\mathrm{K}$ shell is:

$$
E f_{K}=\omega_{K} \cdot\left[1-P_{X K N}-P_{X K O}-P_{X K L 3}\left(1-E f_{L 3}\right)-P_{X K L 2}\left(1-E f_{L 2}\right)\right]+\left(1-\omega_{K}\right)
$$

The detection efficiency of a vacancy in the K shell, $E f_{K}$, is 0.962 .

The detection efficiencies associated with each subshell are resumed in Table 1.

\section{Detection efficiency of electron capture}

The electron capture to the $618 \mathrm{keV}$ excited level of iodine, with a probability of $0.014 \%$ is neglected. The probabilities of electron capture towards the $375 \mathrm{keV}$ and $203 \mathrm{keV}$ excited levels are presented in Table 2.

The calculation of the detection efficiency of the electron capture is the sum of the detection efficiencies for each vacancy, weighed by the probability of capture to the respective shell. This gives the values for the detection efficiencies of capture $E f_{e c 375}=0.960$ and $E f_{\text {ec203 }}=0.960$.

\section{Detection efficiencies of the gamma transitions}

It can be assumed that, the gamma emissions are not detected but the conversion electrons are detected with a probability of 1 . The detection efficiency associated with each gamma transition is thus just related to the conversion coefficients.

The conversion coefficients, relative intensity and associated detection efficiencies of each of the gamma transitions are summarized in Table 3 . The relative intensity is the ratio of the considered gamma transition divided by the sum of the gamma transitions from the parent level.

The detection efficiency of the gamma transition from the $58 \mathrm{keV}$ level is: $E f_{58}=0.792$

The detection efficiency of the gamma transitions from the $203 \mathrm{keV}$ level, $E f_{2203}$ is a combination of the efficiencies due to the transitions $\gamma_{1}, \gamma_{2}$ and $\gamma_{4}$.

$$
E f_{\gamma 203}=I_{\gamma 2}\left(\varepsilon_{\gamma 1}+\left(1-\varepsilon_{\gamma 1}\right) \varepsilon_{\gamma 2}\right)+I_{\gamma 4} \varepsilon_{\gamma 4}
$$

This gives $E f_{\gamma 203}=0.162$.

The detection efficiency of the gamma transitions from the $375 \mathrm{keV}$ level, $E f_{375}$, is a combination of the efficiencies of the gamma transitions from this excited level.

$$
E f_{\gamma 375}=I_{\gamma 3}\left(\varepsilon_{\gamma 3}+\left(1-\varepsilon_{\gamma 3}\right) E f_{203}\right)+I_{\gamma 5} \varepsilon_{\gamma 5}
$$

This gives $E f_{\gamma 375}=0.184$

\section{Detection efficiency of each electron capture decay path}

The detection efficiency of each electron capture path is:

$$
\begin{aligned}
& E f_{e c 2}=E f_{e c 375}+\left(1-E f_{e c 375}\right) E f_{\gamma 375} \\
& E f_{e c 3}=E f_{e c 203}+\left(1-E f_{e c 203}\right) E f_{\gamma 203}
\end{aligned}
$$


This gives $E f_{e c 2}=0.967$ and $E f_{e c 3}=0.967$.

The total detection efficiency is the sum of the global detection efficiency of each capture branch, weighed by the respective probabilities of each capture branch.

$$
E f_{\text {total }}=E f_{e c 3} \cdot P_{e c 3}+E f_{e c 2} \cdot P_{e c 2}
$$

As $P_{e c 3}=0.527$ and $P_{e c 2}=0.473$, this gives $E f_{\text {total }}=0.967$.

The uncertainty of this detection efficiency can be calculated from the composition of the uncertainties on each parameter used in the calculation and on the probability of detection of the L X-rays calculated using the Monte Carlo program. This is a difficult task because most of the values used are from calculations and are correlated, but there is no information on the covariances, except the ones that can be derived from balance equation, e.g. when the sum of the parameters of concurrent phenomena is equal to 1 . A way to simplify this evaluation is to only consider the parameters with the higher sensitivity coefficients and the higher uncertainties. As the probabilities of each electron capture decays are similar, and as the corresponding detection efficiencies are also similar, the uncertainties on the electron capture probabilities do not contribute to the global uncertainty on detection efficiency. It can also be observed that the detection efficiency of vacancies in $\mathrm{K}, \mathrm{L}$ an $\mathrm{M}$ shells are similar and very high, but not the one for the $\mathrm{N}$ vacancies. The probability of $\mathrm{N}$ electron capture is 0.0065 (3). The influence of this parameter on the global detection efficiency is thus very small. The uncertainties of the gamma emission and of the conversion coefficients are the dominant components, because this influences the relative intensities of gamma photons, which are not detected, and conversion electrons, which are detected with a probability of 1 . The dominant parameters are the conversion coefficients of $\gamma_{2}$ (relative uncertainty of $0.7 \%$ ) and $\gamma_{3}$ (relative uncertainty of $1.4 \%$ ). Because of the low intensity of $\gamma_{2}$, the only significant contribution is from $\gamma_{4}$, giving a relative uncertainty of $0.9 \%$ to the detection efficiency of the $203 \mathrm{keV}$ level. As the efficiency from $\gamma_{4}$ is weighed by the non-detection probability of the electron capture to the 203-keV level, which is 0.032 , the contribution to the global uncertainty is also negligible.

Eventually, the uncertainty calculations on the probability of detection of LX rays have to be considered. As it is difficult to evaluate the uncertainty on a simulation, we vary this probability of detection by a conservative value of $10 \%$, but this does not change significantly the global detection efficiency of ${ }^{127} \mathrm{Xe}$.

From this we can conclude that the calculation of the detection efficiency is quite robust.

Given all these considerations, we adopted a conservative value of the relative standard uncertainty on the detection efficiency of $0.4 \%$.

\subsection{Results}

The activity per unit volume STP obtained by dividing the counting rate per unit volume STP by the calculated detection efficiency was: $452.1(3.1) \mathrm{Bq} . \mathrm{cm}^{-3}$ at the reference date of 29/12/2013, $12 \mathrm{~h} 00$ UTC. The relative standard uncertainty components include the counting statistics $(0.38 \%)$, the counter volumes $(0.23 \%)$, the reference volume of the gas sample and its STP correction $(0.3 \%)$, the decay corrections to the reference date and the decay correction during the measurement $(0.05 \%)$, the counting uncertainty due to the discriminator threshold adjustments $(0.15 \%)$ and the uncertainty on the detection efficiency $(0.4 \%)$. This reference activity was used for the gamma-rays measurements.

\section{Photon emission intensities}




\subsection{Experimental set-up and calibration}

Gamma-spectrometry measurements were performed using a $100 \mathrm{~cm}^{3}$ n-type high-purity germanium (HPGe) detector linked to analog electronics. Twenty radionuclides were used to establish the efficiency calibration, with point sources prepared from standard solutions, with activity per unit mass given with relative uncertainties lower than $0.5 \%$. The experimental data (energy, efficiency) were fitted using a log-log polynomial function. The efficiency calibration, for point sources at $10.2 \mathrm{~cm}$ from the detector window, is obtained over the 15 to $1836 \mathrm{keV}$ energy range with a relative combined standard uncertainty of $0.5 \%$ for the high energy part, and of 1 to $2 \%$ below $100 \mathrm{keV}$. Since this calibration was established for geometrical conditions different from the gas geometry, calculation of efficiency transfer factors was necessary, using the code ETNA (Piton et al., 2000).

\subsection{Gas sample preparation}

The sample of gas was conditioned in a cylindrical stainless steel container with external diameter and height equal to $5.4 \mathrm{~cm}$. The bottom thickness was obtained owing to ultrasonic measurement and found to be $1.54 \mathrm{~mm}$. The container volume was equal to $(100.04 \pm 0.88) \mathrm{cm}^{3}$. The gas was sampled from the xenon standard source under controlled pressure and temperature conditions. Its activity $A$ was derived from the activity per unit volume and the STP volume of the sample. Finally, the sample activity at the reference date was $12.580(140) \mathrm{kBq}$. The container was installed on a specific support fitted onto the detector end cap, its bottom being then located at a distance of $6.83 \mathrm{~cm}$ from the detector window.

\subsection{Impurities checking}

Complementary measurements were performed using a $5 \mathrm{~cm}^{3}$ glass vial that allowed the $\mathrm{X}$ rays to exit from the container and to be registered in the detector. This measurement was also used to check the possible impurities of ${ }^{125} \mathrm{I}$ and ${ }^{129} \mathrm{I}$. In their decay, both radionuclides emit low energy gamma-rays, respectively $35.49 \mathrm{keV}$ and $39.58 \mathrm{keV}$, close to the $\mathrm{K} \mathrm{X}$-ray lines of iodine resulting from the ${ }^{127} \mathrm{Xe}$ decay. In this measurement, the detection limit for both radionuclides relative to the ${ }^{127} \mathrm{Xe}$ activity was $5.10^{-3}$.

\subsection{Spectrum processing}

The processing of the ${ }^{127}$ Xe spectrum is quite straightforward in the gamma energy range, and the relevant peak areas, $S(E)$, were determined by Gaussian fit, after subtraction of a step-like function for the background (Ruellan et al., 1996). For each energy, $E$, the photon emission intensity, $I(E)$, was calculated as:

$$
I(E)=\frac{S(E) \cdot C_{D} \cdot C_{M} \cdot C_{C}(E)}{A \cdot t . \varepsilon(E) \cdot C_{G}(E)}
$$

where $A$ is the sample activity, $t$ is the acquisition live-time, $\varepsilon(E)$ is the full-energy peak efficiency for photons with energy $E$, emitted from a point source (reference conditions), $C_{D}$ is the decay correction factor, $C_{M}$ is the correction factor for the decay during measurement, $C_{C}(E)$ is the correction factor for coincidence summing (Ferreux et al., 2012), $C_{G}(E)$ is the correction factor for geometry transfer from the reference efficiency calibration to the measurement condition. 


\subsection{Results}

Absolute photon emission intensities of ${ }^{127} \mathrm{Xe}$ are derived and presented in Table 4. Six gamma-ray lines were measured with relative combined standard uncertainties ranging from 1.3 to $7.7 \%$. Relative values are also given, the emission intensity of the $202 \mathrm{keV}$ line being taken as reference with $100 \%$ intensity. The K X-ray emission intensity values could also be derived in relative value: these were obtained from the glass vial measurement (see §3.3) and obtained using the $57.61 \mathrm{keV}$ relative emission intensities as transfer value, the associated relative standard uncertainties being $2.3 \%$. Details of uncertainty components are given in Table 5.

\section{Discussion}

Comparison of the $\gamma$-ray emission intensities measurements published in the literature with this work can be found in Table 4.

Data from (Collé and Kishore, 1974) were given as absolute intensity measurements. The efficiency calibration was performed using standard calibrated sources, but no activity measurement of the ${ }^{127} \mathrm{Xe}$ source was given in the publication. For this reason we chose not to use these results for the absolute intensity of the 203-keV transition as a reference to evaluate the other $\gamma$-ray intensities.

The data from (Gehrke and Helmer, 1977) and from this work are absolute intensity measurements. The efficiency calibration was performed using standard calibrated sources and an activity measurement of the ${ }^{127}$ Xe source is provided in each case.

Consequently, only (Gehrke and Helmer, 1977) and this work were conserved to evaluate the absolute $\gamma$ intensity of the $203 \mathrm{keV}$ emission. The data are consistent. A weighted average using its internal uncertainty, with a reduced $\chi^{2}$ of 1.4 , gives: $I_{\gamma}(203 \mathrm{keV})=68.45(45) \%$.

The adopted energies of the ${ }^{127} \mathrm{I}$ levels are from (Hashizume, 2011). Mixing ratios and multipolarities were evaluated with available literature and these parameters were used to calculate the internal conversion coefficients $\alpha_{\mathrm{K}}$ and $\alpha_{\mathrm{T}}$ with the BrIcc program (Kibédi et al., 2008). Consistency of the decay scheme is tested summing all the $\gamma$-rays falling to the ground state of

${ }^{127}$ I. This sum is equal to: $\Sigma P_{\gamma+c e}=99.8$ (14) \%.

From these absolute emission intensities, the following probabilities of electron-capture transitions were found:

$$
P_{\varepsilon}(0,2)=52.7(15) \%, P_{\varepsilon}(0,3)=47.3(7) \%, P_{\varepsilon}(0,4)=0.0142(9) \% .
$$

The resulting decay scheme is resumed in Figure 1.

\section{Conclusion}

We showed in this study that the standardization of ${ }^{127}$ Xe can be achieved using differential internal proportional counter measurements, with a relative combined standard uncertainty of $0.7 \%$. The detection efficiency is not very dependent on the decay scheme and this allows the use of the activity of this standard for the determination of the gamma-ray emission intensities. The better knowledge of the absolute gamma emission intensity lead to the conclusion that activity measurements of ${ }^{127} \mathrm{Xe}$ by gamma spectrometry can be made with relative combined standard uncertainties lower than $2 \%$. From this we can confirm that ${ }^{127} \mathrm{Xe}$ 
can be useful in the quality control of remote radio-xenon monitoring stations, with the advantage of a much longer half-life than ${ }^{133} \mathrm{Xe}$ or ${ }^{131 \mathrm{~m}} \mathrm{Xe}$.

\section{References}

Chen, H. M., Crasemann B., 1979. Relativistic radiationless transition probabilities for atomic K- and L-shells. Atom. Data Nucl. Data Tables, vol. 24, issue 1, pp. 13-37.

Collé, R., Kishore, R., 1974. Absolute $\gamma$-Ray Intensities in the Decay of ${ }^{79} \mathrm{Kr}$ and ${ }^{127} \mathrm{Xe}$. Phys. Rev. C9, 981

Forrest, R.N., Easterday, H.T., 1958. Radiations of Xe-127. Phys. Rev. 112, 950

Gehrke, R.J., Helmer, R.G., 1977. Absolute gamma-Ray Intensities from 127Xe Decay. Int. J. Appl. Radiat. Isotop. 28, 744

Geiger, J.S., 1967. Decay-Scheme Studies of ${ }^{125}$ Xe and ${ }^{127}$ Xe. Phys. Rev. 158, 1094

Hashizume, A., 2011. Nuclear Data Sheets for A = 127. Nucl. Data Sheets 112, 1647

Kibédi, T., Burrows, T.W., Trzhaskovskaya, M.B., Davidson, P.M., Nestor, C.W. Jr., 2008.

Evaluation of theoretical conversion coefficients using BrIcc. Nucl. Instr. and Meth. A589, 202-229

Lansiart, A., Dhilly, G., Picolo, J. L., 1993. Note Technique LPRI/93/010.

Lépy, M.-C, Ferreux, L., Pierre, S., 2012. Coincidence summing corrections applied to volume sources, Applied Radiation and Isotopes, 70 (2012) 2137-2140.

McGuire, J. Atomic M-shell Coster-Kronig, (1972). Auger, and radiative Rates and Fluorescence Yields for Ca-Th. Phys Rev A 5,3, 1043

McGuire, J. Atomic N-shell Coster-Kronig, (1974). Auger, and radiative Rates and Fluorescence Yields for $38 \leq Z \leq 103$. Phys Rev A 9,5, 1840

Piton, F., Lépy, M.-C, Bé, Plagnard, J., 2000. Efficiency transfer and coincidence summing corrections for gamma-ray spectrometry. Applied Radiation and Isotopes 52, 791-795.

Puri, S., Mehta, D., Chand, B., Nirmal Singh and Trehan, P. N., 1993. L shell fluorescence yields and Coster-Kronig transition probabilities for the elements with $25 \leqslant Z \leqslant 96$. X-Ray Spectrometry Volume 22, Issue 5, 358-361.

Ruellan, H., Lépy, M.-C., Etcheverry, M., Morel, J., 1996. A new spectra processing code applied to the analysis of ${ }^{235} \mathrm{U}$ and ${ }^{238} \mathrm{U}$ in the 60 to $200 \mathrm{keV}$ energy range. Nucl. Instrum. Meth. in Phys. Res. A369, 651-656. 
Salvat, F., Fernandez-Varea, J.M., Sempau, J., 2009. PENELOPE-2008: A code system for Monte Carlo Simulation of Electron and Photon transport, OECD Nuclear Energy Agency, Issy-les-moulineaux, France.

Schmidt-Ott, W.-D., Weirauch, W., Smend, F., Langhoff, H., Gfoller, D., 1968. Der Zerfall des $125 \mathrm{mXe}$, des 127mXe und des 127gXe. Z. Physik 217, 282

Scofield J. H., 1974. Relativistic Hartree-Slater values for K and L X-ray emission rates. Atom. Data Nucl. Data Tables, vol. 14, pp. 121-137.

Vatin, R., Chauvenet, B., 1987. Direct measurement of xenon-127 activity with a $4 \pi-\gamma$ detector. Appl. Radiat. Isot. 38 (10), 767-770. 


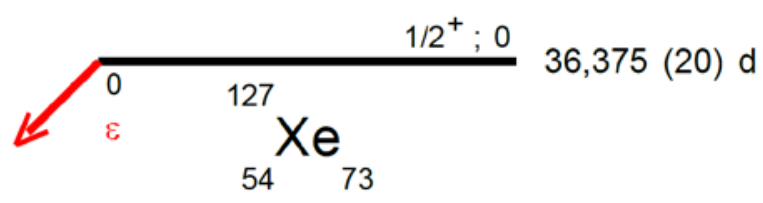

$\gamma$ Emission intensities

per 100 disintegrations

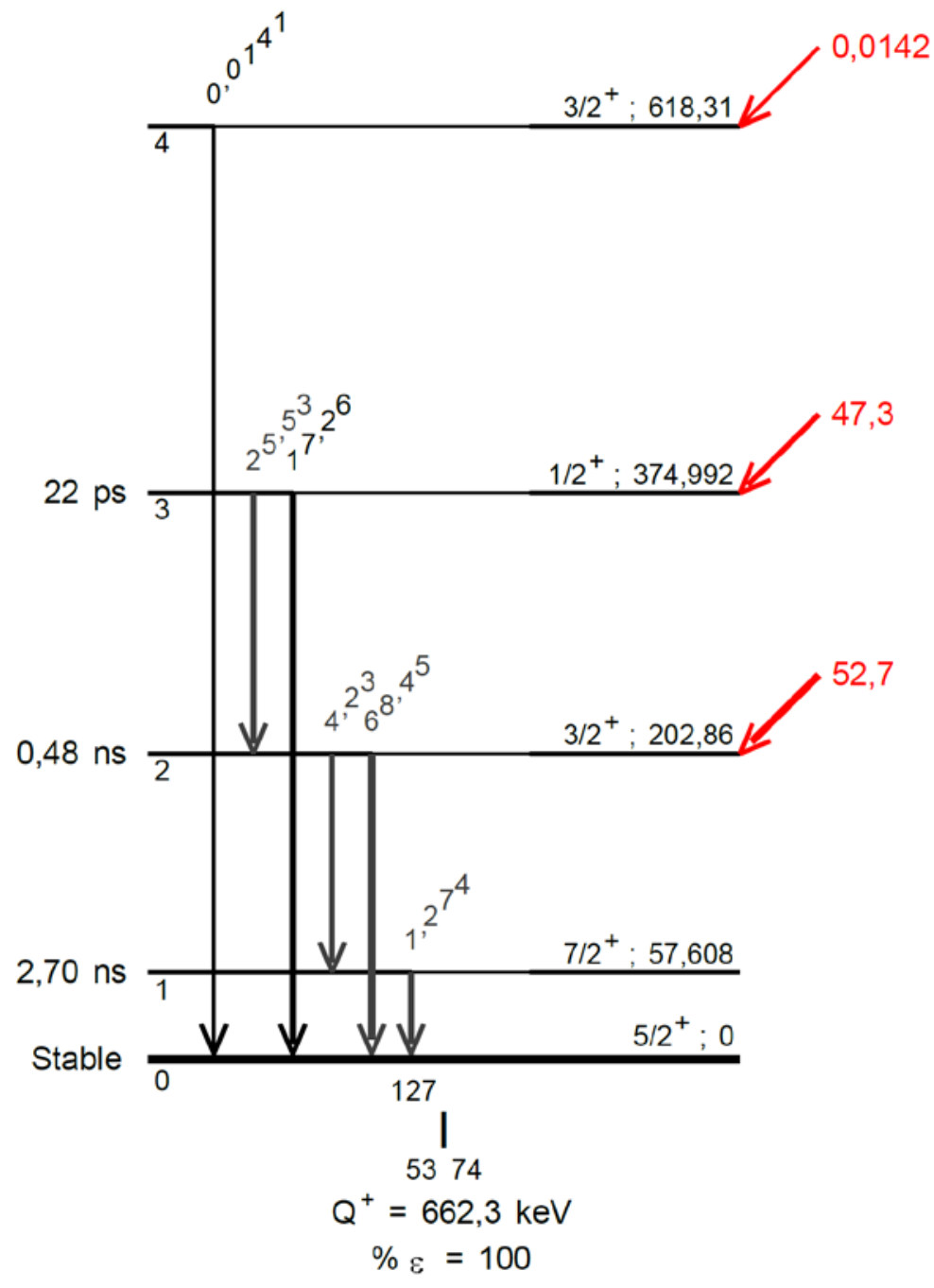

Figure $1:{ }^{127}$ Xe decay scheme 


\begin{tabular}{|c|c|}
\hline Vacancy & Detection efficiency, $E f_{i}$ \\
\hline K shell & 0.9624 \\
\hline $\mathrm{L}_{1}$ shell & 0.982 \\
\hline $\mathrm{L}_{2}$ shell & 0.980 \\
\hline L3 shell & 0.989 \\
\hline M shell & 1 \\
\hline N shell & 0 \\
\hline
\end{tabular}

Table 1: Detection efficiencies for vacancies in each atomic shell

\begin{tabular}{|c|c|c|}
\hline Electron capture probability & Towards 375 keV level & Towards 203 keV level \\
\hline $\mathrm{P}_{\mathrm{K}}$ & 0.834 & 0.8420 \\
\hline $\mathrm{P}_{\mathrm{L} 1}$ & 0.104 & 0.098 \\
\hline $\mathrm{P}_{\mathrm{L} 2}$ & 0.029 & 0.027 \\
\hline $\mathrm{P}_{\mathrm{M}}$ & 0.027 & 0.027 \\
\hline $\mathrm{P}_{\mathrm{N}}$ & 0.006 & 0.006 \\
\hline
\end{tabular}

Table 2: Electron capture probabilities

\begin{tabular}{|c|c|c|}
\hline Gamma transition & $\alpha_{\mathrm{t}}$ & Detection efficiency, $\varepsilon_{\gamma i}$ \\
\hline $1(1-0)$ & 3.72 & 0.792 \\
\hline $2(2-1)$ & 0.471 & 0.321 \\
\hline $3(3-2)$ & 0.165 & 0.144 \\
\hline $4(2-0)$ & 0.113 & 0.104 \\
\hline $5(3-0)$ & 0.0199 & 0.020 \\
\hline
\end{tabular}

Table 3: Conversion coefficients and detection efficiencies of ${ }^{127}$ Xe gamma transitions 


\begin{tabular}{|c|c|c|c|c|c|c|c|c|}
\hline & \multicolumn{3}{|c|}{ Absolute emission intensities } & \multicolumn{5}{|c|}{ Relative emission intensities } \\
\hline $\mathrm{E}(\mathrm{keV})$ & This work & $\begin{array}{c}\text { Collé and } \\
\text { Kishore, } \\
1974\end{array}$ & $\begin{array}{c}\text { Gehrke } \\
\text { and } \\
\text { Helmer, } \\
1977 \\
\end{array}$ & This work & $\begin{array}{c}\text { Forrest and } \\
\text { Easterday, } \\
1958\end{array}$ & $\begin{array}{c}\text { Geiger }^{1} \text {, } \\
1967\end{array}$ & $\begin{array}{c}\text { Collé and } \\
\text { Kishore }^{2} \text {, } \\
1974\end{array}$ & $\begin{array}{c}\text { Gehrke } \\
\text { and } \\
\text { Helmer }^{2} \text {, } \\
1977 \\
\end{array}$ \\
\hline $\mathrm{X} \mathrm{K} \alpha$ & & & & $83.4(17)$ & \multirow{2}{*}{$117(7)$} & & & \\
\hline $\mathrm{X} \mathrm{K} \beta$ & & & & $20.35(42)$ & & & & \\
\hline 57 & $1.23(7)$ & $1.31(8)$ & $1.22(7)$ & $1.76(10)$ & $2.1(2)$ & $1.91(9)$ & $1.92(12)$ & $1.79(10)$ \\
\hline 145 & $4.33(7)$ & $4.24(21)$ & $4.29(13)$ & $6.20(13)$ & $6.4(10)$ & $6.06(17)$ & $6.23(33)$ & $6.28(19)$ \\
\hline 172 & $26.23(44)$ & $24.7(1)$ & $25.5(8)$ & $37.6(8)$ & $34.2(17)$ & $37.3(10)$ & $36.3(16)$ & $37.3(12)$ \\
\hline 203 & $69.8(12)$ & $68.1(13)$ & $68.3(4)$ & 100.0 & 100 & 100.0 & 100.0 & 100.0 \\
\hline 375 & $17.32(28)$ & $17.4(1)$ & $17.2(5)$ & $24.8(5)$ & $30.1(12)$ & $26.2(8)$ & $25.6(15)$ & $25.2(7)$ \\
\hline 618 & $\begin{array}{c}0.0147 \\
(20)\end{array}$ & & $0.014(1)$ & $\begin{array}{c}0.0211 \\
(29)\end{array}$ & & & & $\begin{array}{c}0.0205 \\
(15)\end{array}$ \\
\hline
\end{tabular}

1. From relative electron data, calculated using the $\alpha_{\mathrm{K}}$ from BrIcc (see text)

2. Relative data calculated from the absolute values

Table 4: Comparison of the photon emission intensities measurements

\begin{tabular}{|l|c|}
\hline & $\begin{array}{c}\text { Relative standard uncertainty } \\
(\%)\end{array}$ \\
\hline Source position & 0.26 \\
\hline Peak area (statistics) & $0.06(202 \mathrm{keV})$ to $7.6(618 \mathrm{keV})$ \\
\hline Peak area determination & 0.2 \\
\hline Efficiency calibration & $0.5(\mathrm{E}>100 \mathrm{keV})$ to 2 \\
\hline Correction for decay & 0.05 \\
\hline Correction for acquisition time & 0.002 \\
\hline Correction for coincidence summing & 0.1 \\
\hline Correction for efficiency transfer & 0.5 to 5 \\
\hline Combined standard uncertainty & 1.4 to 12.3 \\
\hline
\end{tabular}

Table 5: Uncertainty budget on the absolute photon emission intensities 